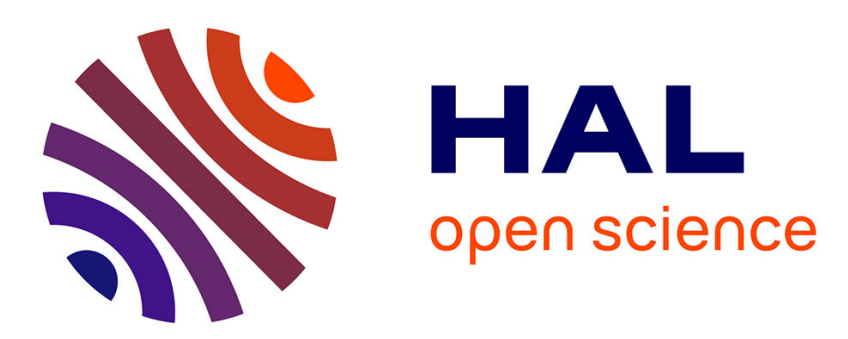

\title{
Edgeworth expansions and rates of convergence for normalized sums: Chung's 1946 method revisited
}

\author{
Helmut Finner, Thorsten Dickhaus
}

\section{To cite this version:}

Helmut Finner, Thorsten Dickhaus. Edgeworth expansions and rates of convergence for normalized sums: Chung's 1946 method revisited. Statistics and Probability Letters, 2010, 80 (23-24), pp.1875. 10.1016/j.spl.2010.08.014 . hal-00691779

\section{HAL Id: hal-00691779 \\ https://hal.science/hal-00691779}

Submitted on 27 Apr 2012

HAL is a multi-disciplinary open access archive for the deposit and dissemination of scientific research documents, whether they are published or not. The documents may come from teaching and research institutions in France or abroad, or from public or private research centers.
L'archive ouverte pluridisciplinaire HAL, est destinée au dépôt et à la diffusion de documents scientifiques de niveau recherche, publiés ou non, émanant des établissements d'enseignement et de recherche français ou étrangers, des laboratoires publics ou privés. 


\section{Accepted Manuscript}

Edgeworth expansions and rates of convergence for normalized sums:

Chung's 1946 method revisited

Helmut Finner, Thorsten Dickhaus

PII:

S0167-7152(10)00237-3

DOI:

10.1016/j.spl.2010.08.014

Reference: $\quad$ STAPRO 5773

To appear in: Statistics and Probability Letters

Received date: 19 August 2009

Revised date: 28 May 2010

Accepted date: 17 August 2010

Please cite this article as: Finner, H., Dickhaus, T., Edgeworth expansions and rates of convergence for normalized sums: Chung's 1946 method revisited. Statistics and Probability Letters (2010), doi:10.1016/j.spl.2010.08.014

This is a PDF file of an unedited manuscript that has been accepted for publication. As a service to our customers we are providing this early version of the manuscript. The manuscript will undergo copyediting, typesetting, and review of the resulting proof before it is published in its final form. Please note that during the production process errors may be discovered which could affect the content, and all legal disclaimers that apply to the journal pertain. 


\title{
Edgeworth expansions and rates of convergence for normalized sums: Chung's 1946 method revisited
}

\author{
Helmut Finner ${ }^{\mathrm{a}}$, Thorsten Dickhaus, ${ }^{* \mathrm{~b}}$ \\ ${ }^{a}$ Institute of Biometrics and Epidemiology, German Diabetes Center, Düsseldorf, \\ Germany \\ ${ }^{b}$ Department of Mathematics, Humboldt-University Berlin, Germany
}

\begin{abstract}
In this paper we revisit, correct and extend Chung's 1946 method for deriving higher order Edgeworth expansions with respect to $t$-statistics and generalized self-normalized sums. Thereby we provide a set of formulas which allows the computation of the approximation of any order and specify the first four polynomials in the Edgeworth expansion the first two of which are well known. It turns out that knowledge of the first four polynomials is necessary and sufficient for characterizing the rate of convergence of the Edgeworth expansion in terms of moments and the norming sequence appearing in generalized self-normalized sums. It will be shown that depending on moments and norming sequence the rate of convergence can be $O\left(n^{-i / 2}\right)$, $i=1, \ldots, 4$. Finally, we study expansions and rates of convergence if the normal distribution is replaced by the $t$-distribution.

Key words: Generalized self-normalized sum, Hermite polynomials, normal approximation, statistical algorithms, t-distribution, t-statistic

${ }^{*}$ Corresponding author. Humboldt-University Berlin, Department of Mathematics, Unter den Linden 6, 10099 Berlin, Germany. Tel: +49 3020935841, Fax: +49 3020935848. E-mail address: dickhaus@math.hu-berlin.de (Th. Dickhaus).
\end{abstract}




\section{Introduction}

In 1946, Kai-Lai Chung derived an expansion for the distribution of Student's t-statistic for an iid sequence of real valued random variables $\left(\zeta_{n}: n \in \mathbb{N}\right)$ with $\mathbb{E} \zeta_{1}=0$ and $\operatorname{Var} \zeta_{1}=1$ in terms of the cumulative distribution function (cdf) $\Phi$ and derivatives of the probability density function (pdf) $\varphi$ of the standard normal distribution $\mathcal{N}(0,1)$, cf. Chung (1946). Thereby the t-statistic is defined by $S_{n}=\sqrt{n} \bar{\zeta} / s$ with $s=\left(n^{-1} \sum_{i=1}^{n}\left(\zeta_{i}-\bar{\zeta}\right)^{2}\right)^{1 / 2}$ and $\bar{\zeta}=\sum_{i=1}^{n} \zeta_{i} / n$. In modern notation, the expansion for the cdf $F_{n}$ (say) of $S_{n}$ derived by Chung (1946) can be written as

$$
F_{n}(x)=\Phi(x)+\varphi(x) \sum_{i=1}^{r} n^{-i / 2} q_{i}(x)+o\left(n^{-r / 2}\right) \text { uniformly in } x \in \mathbb{R}
$$

and is called an Edgeworth expansion. It is known to be valid if $\mathbb{E}\left|\zeta_{1}\right|^{r+2}<\infty$ for some integer $r \geq 1$ and the distribution of $\zeta_{1}$ has a nontrivial absolutely continuous component with respect to the Lebesgue measure (cf. Hall (1987)). Each $q_{i}$ appearing in this formula is a polynomial of order $3 i-1$ the coefficients of which depend on the moments $\alpha_{j}=\mathbb{E} \zeta_{1}^{j}, j=3, \ldots, i+2$.

Unfortunately, the explicit expansion given in equation (35) in Chung (1946) is incorrect as noted earlier in Wallace (1958) and to our knowledge there seems to be no published correction. Once the well-hidden error in Chung (1946) is corrected, the computation of the first polynomials $q_{i}$ appearing in (1) is elementary and straightforward with Chung's method. In Section 2 we extend the formulas given in Chung (1946) such that in principle the $q_{i}$ 's are computable up to arbitrary order with an algebraic computer package. 
Alternatively, the $q_{i}$ 's can be computed as outlined in Hall (1992) under a broad scope of so-called 'smooth function models'. While this method applies to many other statistics, the computational effort of this method seems to be more tedious for self-normalized sums than Chung's method. Anyhow, the first two polynomials $q_{1}, q_{2}$ appearing in the expansion (1) are computed for example in Hall (1987), Hall (1992) and can be found in various textbooks. They are given by $q_{1}(y)=\left(y^{2} / 3+1 / 6\right) \alpha_{3}$ and $q_{2}(y)=$ $\left(y^{3} / 12-y / 4\right) \alpha_{4}-\left(y^{5} / 18+y^{3} / 9-y / 6\right) \alpha_{3}{ }^{2}-y^{3} / 2$. This representation shows that the rate of convergence is $O\left(n^{-1 / 2}\right)$ in case of $\alpha_{3} \neq 0$ and $O\left(n^{-1}\right)$ in case of $\alpha_{3}=0$. Obviously, the best possible rate of convergence is $O\left(n^{-1}\right)$ and this may be the reason that usually only the first two polynomials are reported.

In Lehmann and Romano (2005), Section 11.4.2, an Edgeworth expansion for the classical $t$-statistic with normalization $(n-1)^{-1}$ in the definition of $s$ is given. The approximation polynomials in this case differ from the $q_{i}$ 's in (1). Hence, the norming sequence in the denominator of a self-normalized sum is of importance for the asymptotic behavior of these $t$-type statistics. This observation provokes the question whether there exist other norming sequences for specific values of the moments $\alpha_{i}, i \geq 3$, such that the rate of convergence can be improved. It will be shown in Section 3 that the answer is positive and that the first four polynomials $q_{1}, \ldots, q_{4}$ suffice to characterize the rate of convergence.

Section 4 is concerned with deriving Edgeworth-type expansions for and analyzing rates of convergence of self-normalized sums if the $t$-distribution is used as approximating distribution instead of the standard normal. 


\section{Chung's method revisited}

Many of the derivations in Chung (1946) refer to formulas and definitions taken from Hsu (1945). Here we restrict attention to the ingredients which suffice to compute the polynomials $q_{i}$ in (1). We correct the main inaccuracy in Chung (1946) and extend the formulas where necessary. However, since we have to omit some technical details of Chung's method here, it can only fully be understood by reading Chung (1946), parts of Hsu (1945) and this paper simultaneously.

In the derivations in Chung (1946) the function $g$ defined by $g(\lambda)=$ $z\left(1+\lambda^{2} z^{2}\right)^{-1 / 2}\left[1+\sum_{j=1}^{\infty} \Gamma(3 / 2) / \Gamma(3 / 2-j) / \Gamma(j+1)\left(\alpha_{4}-1\right)^{j / 2}(\lambda x)^{j}\right]$ and its derivatives $g^{(i)}$ play a crucial role. The formulas given in Chung (1946), p. 458 , fail by introducing the abbreviation $z^{\prime}=z\left(1+\lambda^{2} z^{2}\right)^{-1 / 2}$ and ignoring that $z^{\prime}$ depends on $\lambda$. The correct derivatives $g^{(i)}(0), i=1, \ldots, 4$, in $\lambda=0$ are given in Appendix A.

A further important quantity in Chung (1946) is $h(\zeta)=t_{1}\left(\zeta^{2}-1\right) /\left(\alpha_{4}-\right.$ $1)^{1 / 2}+t_{2} \zeta$, whereby $\zeta$ has the same distribution as $\zeta_{1}$. Let $U_{j}\left(t_{1}, t_{2}\right)$ denote the $j$ th cumulant of $h(\zeta)$ and define $m_{k}\left(t_{1}, t_{2}\right)=-\sum_{\ell=0}^{k-3} i^{\ell+1} U_{\ell+3}\left(t_{1}, t_{2}\right) \lambda^{\ell+1} /(\ell+$ $3)$ ! and $\Psi_{k}\left(i t_{1}, i t_{2}\right)=\sum_{j=1}^{k-3} m_{j}\left(t_{1}, t_{2}\right)^{j} / j$ !. Expanding the $U_{i}$ 's in terms of $t_{1}, t_{2}$ and replacing $\left(i t_{1}\right)^{p}\left(i t_{2}\right)^{q}$ by $(-1)^{p+q} w_{p q}(x, y)$ in $\Psi_{k}\left(i t_{1}, i t_{2}\right)$ yields the representation $\Psi_{k}\left(i t_{1}, i t_{2}\right) \equiv \sum_{j=1}^{k-3} \gamma_{j}(x, y)$, where $\gamma_{j}(x, y)=O\left(\lambda^{j}\right)$, $w_{p q}(x, y)=\partial^{p+q} /\left(\partial x^{p} \partial y^{q}\right) w(x, y)$ appears repeatedly in $\gamma_{j}(x, y)$ for various $p, q$ with $p+q \leq 3 r$ and $w(x, y)=\left(2 \pi\left(1-\rho^{2}\right)^{1 / 2}\right)^{-1} \exp \left(-\left(x^{2}-2 \rho x y+\right.\right.$ $\left.\left.y^{2}\right) /\left(2\left(1-\rho^{2}\right)\right)\right)$. Finally, letting $f_{p q}(\lambda)=\int_{-\infty}^{\infty} \int_{-\infty}^{g(\lambda)} w_{p q}(x, y) d y d x$, the formal approximation in Chung (1946) is given by $F_{n}(z)=\sum_{j=0}^{r} f_{00}^{(j)}(0) / j !+$ $\sum_{j=1}^{r} \int_{-\infty}^{\infty} \int_{-\infty}^{g(\lambda)} \gamma_{j}(x, y) d y d x+o\left(\lambda^{r}\right)$, where $f_{p q}(\lambda)$ is approximated by its 
Taylor series in $\lambda=0$ wherever it appears in $\int_{-\infty}^{\infty} \int_{-\infty}^{g(\lambda)} \gamma_{j}(x, y) d y d x$. In fact, the latter integral can be rewritten in terms of $f_{p q}(\lambda)$, which in turn can be expressed in terms of $I_{p q}^{r}(z)=\int_{-\infty}^{\infty} x^{r} w_{p q}(x, z) d x$. In Chung (1946) non-vanishing $I_{p q}^{r}$ 's needed for the computation of the first three polynomials $q_{1}, q_{2}, q_{3}$ are computed for $p=0, \ldots, 3, r=p, \ldots, 3$. We extend Chung's table for all values of $p, q$ by providing the full recursive regime for the computation of the $I_{p q}$ 's.

Lemma 2.1. The non-vanishing $I_{p q}$ 's are given by the following recursive relationship.

$$
\begin{aligned}
I_{0 q}^{0}(z) & =\varphi^{(q)}(z), I_{0 q}^{1}(z)=-\rho \varphi^{(q+1)}(z), q \geq 0, \\
I_{0 q}^{r+1}(z) & =-\rho I_{0, q+1}^{r}(z)+r I_{0 q}^{r-1}(z), q \geq 0, r \geq 1, \\
I_{p q}^{r}(z) & =-r I_{p-1, q}^{r-1}(z), 1 \leq p \leq r, q \geq 0 .
\end{aligned}
$$

Proof. The equations in (2) are as in Chung (1946) while (3) and (4) can be verified by making use of the definition of $I_{p q}^{r}(z)$.

Remark 2.1. Equation (3) is interesting in itself because it is related to Hermite polynomials with positive coefficients. Let $H_{n}$ denote the normalized Hermite polynomials defined by $H_{1}(x)=x, H_{2}(x)=x^{2}-1, H_{n+1}(x)=$ $x H_{n}(x)-n H_{n-1}(x)$ and define modified Hermite polynomials by $h_{n}(x)=$ $-(I / \sqrt{2})^{n} H_{n}(I x / \sqrt{2})$. Then the coefficients of $h_{n}$ equal the coefficients of $H_{n}$ except that they are all positive. Interestingly, $I_{0 q}^{r}=h_{r}(\rho D)\left(\varphi^{(q)}\right)$, where $D$ denotes the differential operator. Note that (3), that is, $I_{0 q}^{r+1}(z)=-\rho I_{0, q+1}^{r}(z)$ $+r I_{0 q}^{r-1}(z)$ corresponds to $h_{r+1}(x)=-x h_{r}(x)+r h_{r-1}(x)$. Moreover, $I_{0 q}^{r}(z)=$ $\left(\partial^{q} / \partial z^{q}\right) \mathbb{E}\left[X^{r} \mid Z=z\right]$, where $(X, Z)$ are bivariate normally distributed with means 0 , variances 1 and covariance $\rho$. 
The next lemma provides the $f_{p q}^{(j)}(0)$ 's for $j=0, \ldots, 4$, which are essential for the computation of $q_{1}, \ldots, q_{4}$.

Lemma 2.2. Setting $I_{p q}^{r} \equiv I_{p q}^{r}(z)$ for $r=0, \ldots, 4$, we have for $p, q \geq 0$ that $f_{00}(0)=\Phi(z), f_{p q}(0)=-I_{p, q-1}^{0} \mathbf{1}_{\mathbb{N}>0}(q), f_{p q}^{(1)}(0)=z\left(\alpha_{4}-1\right)^{1 / 2} I_{p q}^{1} / 2$, $f_{p q}^{(2)}(0)=\left(\alpha_{4}-1\right)\left(z^{2} I_{p q+1}^{2}-z I_{p q}^{2}\right) / 4-z^{3} I_{p q}^{0}, f_{p q}^{(3)}(0)=-3\left(\alpha_{4}-1\right)^{1 / 2}\left(z^{3} I_{p q}^{1}+\right.$ $\left.z^{4} I_{p, q+1}^{1}\right) / 2+\left(\alpha_{4}-1\right)^{3 / 2}\left(3 z I_{p q}^{3}-3 z^{2} I_{p, q+1}^{3}+z^{3} I_{p, q+2}^{3}\right) / 8, f_{p q}^{(4)}(0)=9 z^{5} I_{p q}^{0}+$ $3 z^{6} I_{p, q+1}^{0}+3\left(\alpha_{4}-1\right)\left(z^{3} I_{p q}^{2}-z^{4} I_{p, q+1}^{2}-z^{5} I_{p, q+2}^{2}\right) / 2+\left(\alpha_{4}-1\right)^{2}\left(-15 z I_{p q}^{4}+\right.$ $\left.15 z^{2} I_{p, q+1}^{4}-6 z^{3} I_{p, q+2}^{4}+z^{4} I_{p, q+3}^{4}\right) / 16$.

Now all ingredients for the computation of the polynomials $q_{3}$ and $q_{4}$ are collected. Computation by hand remains cumbersome. Therefore we prepared a Maple worksheet which allows the computation of the $q_{i}$ 's up to arbitrary order. Due to the structure of the $f_{p q}^{(j)}$ 's, Lemma 2.2 can be extended by utilizing standard symbolic integration methods. Clearly, hardware resources will limit the number of computable $q_{i}$ 's. We report the result for $q_{3}$ and $q_{4}$ in Appendix B.

Remark 2.2. We also computed the $q_{i}$ 's with the method described in Hall (1992) up to order 6 with complete coincidence. The method proposed in Hall (1992) involves the computation of moments of more complicated statistics and seems more time consuming.

\section{Rates of convergence and generalized self-normalized sums}

In case of $\alpha_{3}=0$ we obtain $q_{1}(y) \equiv 0$. This vanishing of $q_{1}$ for vanishing skewness of $\zeta_{1}$ can be interpreted in that way that on the $n^{-1 / 2}$ scale, the 
Edgeworth approximation of the cdf $F_{n}$ of the statistic $S_{n}$ cannot be distinguished from $\Phi$. On the $n^{-1}$ scale, however, there are always detectable differences between the two cdf's, because it is impossible to find a value for $\alpha_{4}$ such that $q_{2}$ becomes the null polynomial. In other words, the rate of convergence of $F_{n}$ towards $\Phi$ can at most achieve $O\left(n^{-1}\right)$.

In the remainder of this section, we will present an approach to improve this rate of convergence for certain values of the moments $\alpha_{j}$. The idea is to utilize a generalized norming sequence $a_{n}$ (say) in the denominator of the $t$-statistic, i.e., we investigate "generalized self-normalized sums" of the type $T_{n}=\sqrt{n} \bar{\zeta} / \sqrt{a_{n} \sum_{i=1}^{n}\left(\zeta_{i}-\bar{\zeta}\right)^{2}}$ instead of $S_{n}$. Hereby, we consider norming sequences $a_{n}$ of the special form

$$
a_{n}=\frac{1}{n\left(1-\sum_{j=1}^{M} C_{j} n^{-j / 2}\right)}
$$

(implying $\lim _{n \rightarrow \infty} n a_{n}=1$ ) for some integer $M$. We now derive a formal expansion for the generalized self-normalized sum $T_{n}$. Our investigations shall lead to an expansion of the form

$$
F_{T_{n}}(t)=\Phi(t)+\sum_{i=1}^{r} n^{-i / 2} \tilde{q}_{i}(t) \varphi(t)+o\left(n^{-r / 2}\right)
$$

if the $(r+2)$-th moment of $\zeta_{1}$ exists. The coefficients of the polynomials $\tilde{q}_{i}$ in (6) depend not only on the cumulants of $\zeta_{1}$, but additionally on the constants $C_{j}$ appearing in the formal description (5) of the norming sequence $a_{n}$. Expansion (6) will prove useful in analyzing optimal rates of convergence for the self-normalized sum $T_{n}$ by choosing optimal norming constants $C_{j}$.

Notice that $T_{n}=S_{n} / b_{n}$ with $b_{n}=\sqrt{n a_{n}}$. Therefore, $F_{T_{n}}(t)=\mathbb{P}\left(T_{n} \leq\right.$ $t)=\mathbb{P}\left(S_{n} \leq b_{n} t\right)=F_{n}\left(b_{n} t\right)$. From (1) we get under the necessary moment 
condition that for given $r \geq 1$ it holds

$$
F_{T_{n}}(t)=\Phi\left(b_{n} t\right)+\sum_{i=1}^{r} n^{-i / 2} q_{i}\left(b_{n} t\right) \varphi\left(b_{n} t\right)+o\left(n^{-r / 2}\right) .
$$

In order to compute the $\tilde{q}_{i}$ 's, we now have to find suitable expansions of $\Phi\left(b_{n} t\right)$ and $\varphi\left(b_{n} t\right)$ in terms of $\Phi(t)$ and $\varphi(t)$. This can be done by noticing that we can express the leading terms on the right-hand side of (7) (which is the expansion for $F_{T_{n}}$ ) by $\Phi\left(b_{n} t\right)+\sum_{i=1}^{r} n^{-i / 2} q_{i}\left(b_{n} t\right) \varphi\left(b_{n} t\right)=\Phi(t)+$ $\varphi(t)\left[h_{n}(t)+\sum_{i=1}^{r} n^{-i / 2} q_{i}\left(b_{n} t\right) g_{n}(t)\right]$, where the auxiliary functions $h_{n}$ and $g_{n}$ are defined by

$$
h_{n}(t)=\left[\frac{\Phi\left(b_{n} t\right)}{\Phi(t)}-1\right] \frac{\Phi(t)}{\varphi(t)}, \quad g_{n}(t)=\varphi\left(b_{n} t\right) / \varphi(t) .
$$

Hence, if we find suitable expansions for $b_{n}, h_{n}(t)$ and $g_{n}(t)$, the work is done, because the $\tilde{q}_{i}$ 's can then simply be obtained by collecting the $n^{-i / 2}$ terms of the expansions of $h_{n}$ and $g_{n}$ and putting them together with the original $q_{i}$ 's, evaluated at the transformed argument values $b_{n} t$. Indeed, the required expansions for $b_{n}$ and $g_{n}(t)$ are simple applications of the Taylor series of the square root and the exponential functions. For the expansion of $h_{n}(t)$, well-known asymptotic expansions for Mills' ratio are needed additionally. We provide the expansions up to order $n^{-2}$ in Appendix C.

Having expanded $h_{n}(t)$ and $g_{n}(t)$ in this manner, we finally obtain the first two $\tilde{q}_{i}$ 's as $\tilde{q}_{1}(t)=\alpha_{3} t^{2} / 3+C_{1} t / 2+\alpha_{3} / 6$ and $\tilde{q}_{2}(t)=3 t C_{1}{ }^{2} / 8+\alpha_{4} t^{3} / 12+$ $\alpha_{3}{ }^{2} t / 6-t^{3} C_{1}^{2} / 8-\alpha_{3}{ }^{2} t^{3} / 9-\alpha_{3}^{2} t^{5} / 18+\alpha_{3} C_{1} t^{2} / 4+t C_{2} / 2-t^{3} / 2-\alpha_{3} C_{1} t^{4} / 6-$ $\alpha_{4} t / 4$. We omit expressions for $\tilde{q}_{i}$ for $i>2$ here because of lack of space.

Remark 3.1. Setting $M=2, C_{1}=0$ and $C_{2}=1$ in (5), we get the studentized sum $\tilde{S}_{n}=\sqrt{n} \bar{\zeta} / \sqrt{(n-1)^{-1} \sum_{i=1}^{n}\left(\zeta_{i}-\bar{\zeta}\right)^{2}}$ and the corresponding ap- 
proximation polynomials are just the ones given in Section 11.4.2 of Lehmann and Romano (2005).

Theorem 3.1. (Rate of convergence for generalized self-normalized sums).

Let $\Delta_{n}(x)=\left|F_{T_{n}}(x)-\Phi(x)\right|$.

(i) If $\alpha_{3} \neq 0$ or $C_{1} \neq 0$, then $\Delta_{n}(x)=O\left(n^{-1 / 2}\right)$.

(ii) If $\alpha_{3}=C_{1}=0$ and $\left(\alpha_{4} \neq 6\right.$ or $\left.C_{2} \neq 3\right)$, then $\Delta_{n}(x)=O\left(n^{-1}\right)$.

(iii) If $\alpha_{3}=C_{1}=0$ and $\alpha_{4}=6$ and $C_{2}=3$ and $\left(\alpha_{5} \neq 0\right.$ or $\left.C_{3} \neq 0\right)$, then $\Delta_{n}(x)=O\left(n^{-3 / 2}\right)$.

(iv) If $\alpha_{3}=C_{1}=0$ and $\alpha_{4}=6$ and $C_{2}=3$ and $\alpha_{5}=C_{3}=0$, then $\Delta_{n}(x)=O\left(n^{-2}\right)$.

Proof. We have to subsequently solve $\tilde{q}_{i}(t) \equiv 0$ for $C_{i}$ and $\alpha_{i+2}$ for $i=1,2,3$. For $i=1$, this is trivial. Plugging in $\alpha_{3}=C_{1}=0$ in $\tilde{q}_{2}(t)$ leads to $\tilde{q}_{2}(t)=\left(\alpha_{4} / 6-1\right) t^{3} / 2+\left(C_{2}-\alpha_{4} / 2\right) t / 2$, hence, to the assertion in case (ii). With $\alpha_{3}=C_{1}=0$ and $\alpha_{4}=6$ and $C_{2}=3$, we obtain $\tilde{q}_{3}(t)=-\alpha_{5} t^{4} / 20-$ $\alpha_{5} t^{2} / 5+t C_{3} / 2-\alpha_{5} / 40$, and part (iii) of the theorem is proven. For the proof of part (iv), we finally set $\alpha_{3}=C_{1}=0, \alpha_{4}=6, C_{2}=3$ and $\alpha_{5}=C_{3}=0$ leading to $\tilde{q}_{4}(t)=\left(3 / 2-\alpha_{6} / 45\right) t^{5}+\left(\alpha_{6} / 18-11 / 4\right) t^{3}+\left(\alpha_{6} / 6+C_{4} / 2-45 / 4\right) t$. Since the coefficients in the latter polynomial are linearly independent, it is impossible to find values for $\left(\alpha_{6}, C_{4}\right)$ such that $\tilde{q}_{4}$ becomes the null polynomial and therefore the assertion of part (iv) follows.

Theorem 3.1 shows that the generalized self-normalized sum $T_{n}$ can lead to a rate of convergence of $\Delta_{n}(x)$ up to $O\left(n^{-2}\right)$, which is an improvement over the approximation quality of $S_{n}$. 
Remark 3.2. As pointed out in Remark 3.1, the studentized sum $\tilde{S}_{n}$ can be generated from $T_{n}$ by setting $C_{i}=0$ for all $i \neq 2$ and $C_{2}=1$. According to Theorem 3.1, it can therefore only achieve a rate of convergence of $O\left(n^{-1}\right)$. $A$ justification for the special role of $C_{2}=3$ can be given by noticing that the norming $a_{n}=(n-3)^{-1}$ leads to variance standardization of $T_{n}$, that is, $\operatorname{VarT}_{n}=1$, if the $\zeta_{i}$ are iid normally distributed as $N(0,1)$. The special role of $\alpha_{4}=6$ in parts (ii) - (iv) of Theorem 3.1 is not clear to us.

Finally, we give an example where Theorem 3.1 (iv) applies.

Example 3.1. Let $\varphi(x \mid \sigma)$ denote a normal density with respect to mean 0 and variance $\sigma^{2}$ and suppose the density $u$ (say) of $\zeta_{1}$ is a mixture of two normal densities, more precisely, let $u(x)=\alpha \varphi\left(x \mid \sigma_{1}\right)+(1-\alpha) \varphi\left(x \mid \sigma_{2}\right)$ with $\sigma_{1}^{2}=(2 \alpha)^{-1}, \sigma_{2}^{2}=(2(1-\alpha))^{-1}$ and $\alpha=(2+\sqrt{2}) / 4$. Then $E \zeta_{1}=E \zeta_{1}^{3}=$ $E \zeta_{1}^{5}=0, E \zeta_{1}^{2}=1$ and $E \zeta_{1}^{4}=6$. Hence, noticing that $E \zeta_{1}^{6}=90$ and setting $C_{1}=C_{3}=0$ and $C_{2}=3$, Theorem 3.1 (iv) applies in this case.

\section{An Edgeworth-type expansion in terms of Student's t-distri-} bution

The investigations in Section 3 can be utilized in a very straightforward way to derive an Edgeworth-type expansion for $T_{n}$ of the form

$$
F_{T_{n}}(t)=F_{t_{\nu}}(t)+\sum_{i=1}^{r} n^{-i / 2} Q_{i}(t) \varphi(t)+o\left(n^{-r / 2}\right)
$$

in terms of Student's $t$-distribution with $\nu=n-1$ degrees of freedom the cdf of which is denoted by $F_{t_{\nu}}$ in formula (8). Note that a $T_{n}$ with norming sequence $a_{n}=(n-1)^{-1}$ and $\zeta_{1} \sim \mathcal{N}(0,1)$ is exactly $t_{\nu}$-distributed. In the 
latter case, $T_{n}$ will be denoted as $t_{\nu}$ in the remainder. Equation (8) gives insight in the asymptotic magnitude of the deviation of the cdf of $T_{n}$ from $F_{t_{\nu}}$. It might be interesting to analyze if a $t$-approximation of $T_{n}$ will lead to an improved rate of convergence in comparison with a normal approximation. Especially, we aim at answering the question if the norming constants $C_{j}$ can be employed to correct for higher-order moment differences of $\zeta_{1}$ compared with a standard normal variate.

In order to derive the $Q_{i}$ 's in (8), we denote by $q_{i}^{*}, i=1, \ldots, k$, the approximation polynomials that arise for $T_{n}=t_{\nu}$ in (6), i.e., the $\tilde{q}_{i}$ 's for special choices $M=2, C_{1}=0$ and $C_{2}=1$ in (5) and by setting the $\alpha_{j}$ 's equal to the (central) moments of the standard normal distribution. By subtracting the resulting expansion from the general expansion for $T_{n}$, we immediately conclude that $Q_{i}(t)=\tilde{q}_{i}(t)-q_{i}^{*}(t), i=1, \ldots, r$. Carrying out these calculations explicitly, we obtain the first four $q_{i}^{*}(t)$ as $q_{1}^{*}(t)=$ $q_{3}^{*}(t) \equiv 0, q_{2}^{*}(t)=-\frac{t}{4}\left(t^{2}+1\right), q_{4}^{*}(t)=-t\left(3 t^{6}-7 t^{4}+19 t^{2}+21\right) / 96$. Consequently, the first two $Q_{i}$ 's are given by $Q_{1}(t)=\tilde{q}_{1}(t), Q_{2}(t)=-\alpha_{3}^{2} / 18 t^{5}-$ $\alpha_{3} C_{1} t^{4} / 6-\left(1 / 4+\alpha_{3}^{2} / 9-\alpha_{4} / 12+C_{1}^{2} / 8\right) t^{3}+\alpha_{3} C_{1} / 4 t^{2}+\left(3 C_{1}^{2} / 8+C_{2} / 2+\right.$ $\left.\alpha_{3}^{2} / 6-\alpha_{4} / 4+1 / 4\right) t$. Considering rates of convergence, it becomes apparent that $Q_{1}$ only vanishes for $\alpha_{3}=C_{1}=0$ and $Q_{2}$ only vanishes if additionally $\alpha_{4}=3$ and $C_{2}=1$, i.e., the first four moments of $\zeta_{1}$ coincide with a standard normal variate and the first two norming constants $C_{1}$ and $C_{2}$ are chosen as in the classical $t$-distribution case. As stated in Corollary 4.1 below, analogous requirements are also necessary for vanishing polynomials $Q_{3}$ to $Q_{8}$. As a conclusion, utilizing the $t$-approximation (8) instead of the normal approximation (6) does not help to increase rates of convergence, since norming 
cannot be used to compensate for moment differences.

One could try to substitute $\varphi$ in (8) by the pdf $f_{t_{\nu}}$ of the $t_{\nu}$-distribution, i.e., consider an expansion of the form

$$
F_{T_{n}}(t)=F_{t_{\nu}}(t)+\sum_{i=1}^{r} n^{-i / 2} \tilde{Q}_{i}(t) f_{t_{\nu}}(t)+o\left(n^{-r / 2}\right) .
$$

Closely related expressions for $F_{T_{n}}$ for fixed $n$ have already been investigated in 1949 by A. K. Gayen in Gayen (1949) based on M. S. Bartlett's paper Bartlett (1935) by first deriving expressions for the corresponding pdf $f_{T_{n}}$.

In order to derive explicit expressions for the first four $\tilde{Q}_{i}(t)$ 's in (9), we make use of the expansion $\varphi(t)=f_{t_{\nu}}(t)\left[1+\left(1+2 t^{2}-t^{4}\right) /(4 n)+O\left(n^{-2}\right)\right]$. Substituting the latter expansion in (8) leads to $\tilde{Q}_{i} \equiv Q_{i}, i=1,2$, and $\tilde{Q}_{i}(t)=Q_{i}(t)+Q_{i-2}(t)\left(1+2 t^{2}-t^{4}\right) / 4, i=3,4$. Unfortunately, we could only reproduce Gayen's (1949) results up to order $n^{-1}$. Taking limits $(n \rightarrow \infty)$ in Gayen's paper also yields $\tilde{Q}_{i} \equiv Q_{i}, i=1,2$. However, the expressions of order $O\left(n^{-3 / 2}\right)$ associated with the factors $\alpha_{3}^{3}$ and $\alpha_{3} \alpha_{4}$ seem to be in error in Gayen (1949), p. 359, and also taking limits $(n \rightarrow \infty)$ in these expressions does not coincide with our result. Anyhow, we recomputed the original approximation method by Bartlett (cf. Bartlett (1935)) which underlies Gayen's (1949) calculations and reproduced the $\tilde{Q}_{i}$ 's for $i=1, \ldots, 4$.

Utilizing Chung's method, the derivations in Sections 3 and 4 and higher order expansions for the ratio $\varphi(t) / f_{t_{\nu}}(t)$, we calculated the polynomials $Q_{i}$ and $\tilde{Q}_{i}$ up to order 8 . Therewith, we can finally summarize our results regarding rates of convergence of $\left|F_{T_{n}}-F_{t_{\nu}}\right|$ as follows. To this end, denote the $k$-th moment of $\mathcal{N}(0,1)$ by $\alpha_{k}^{*}$ and let $\Delta \alpha_{k}=\alpha_{k}^{*}-\alpha_{k}$, where $\alpha_{k}$ denotes the $k$ th moment of $\zeta_{1}$. Moreover, let $C_{2}^{*}=1, C_{k}^{*}=0$ for $k \neq 2$ and $\Delta C_{k}=C_{k}^{*}-C_{k}$, 
where the $C_{k}$ 's denote the norming constants in the denominator of $T_{n}$ as appearing in the definition of $a_{n}$ in (5).

Corollary 4.1. Assume that the $(M+2)$-nd moment $\alpha_{M+2}$ of $\zeta_{1}$ is finite for some integer $1 \leq M \leq 8$ and the distribution of $\zeta_{1}$ has a nontrivial absolutely continuous component with respect to the Lebesgue measure. Then $\left|F_{T_{n}}-F_{t_{\nu}}\right|=O\left(n^{-k^{*} / 2}\right)$, where $k^{*}=\min \left\{k \in\{1, \ldots, M\}: \Delta \alpha_{k+2} \neq 0 \vee\right.$ $\left.\Delta C_{k} \neq 0\right\}$. If no such $k^{*}$ exists, then $\left|F_{T_{n}}-F_{t_{\nu}}\right|=o\left(n^{-M / 2}\right)$.

Since each polynomial $Q_{i}$ or $\tilde{Q}_{i}$, respectively, only depends on $\alpha_{j}, j=3, \ldots$, $i+2$ and $C_{j}, j=1, \ldots, i$, and equations (8), (9) are valid for $T_{n}=t_{\nu}$, it is clear that also for $M>8$ the conditions

$$
\Delta \alpha_{i+2}=0 \wedge \Delta C_{i}=0 \text { for all } i=1, \ldots, M
$$

imply $Q_{i}(t) \equiv 0$ and $\tilde{Q}_{i}(t) \equiv 0$ for all $i=1, \ldots, M$, i. e., conditions (10) are sufficient for vanishing polynomials up to the $M$-th. We conjecture that conditions (10) are also necessary conditions for any $M \geq 1$ as stated in Corollary 4.1 for $1 \leq M \leq 8$.

Remark 4.1. Maple worksheets for computing the polynomials appearing in the Edgeworth expansions investigated in this paper are available at http: //www. helmut-finner. de. One worksheet computes the polynomials $q_{i}$ with Chung's 1946 method up to arbitrary order. A further worksheet computes these polynomials for $i \leq 5$ with the method described in Hall (1992) based on a smooth function approach. Finally, as an additional crosscheck we provide a worksheet based on the Bartlett-Gayen method for the polynomials $\tilde{Q}_{i}$ with norming sequence $(n-1)^{-1}$. 


\section{References}

Bartlett, M. S., 1935. The effect of non-normality on the $t$ distribution. Proc. Camb. Philos. Soc. 31, 223-231.

Chung, K.-L., 1946. The approximate distribution of Student's statistics. Ann. Math. Stat. 17, 447-465.

Gayen, A. K., 1949. The distribution of 'Student's' $t$ in random samples of any size drawn from non-normal universes. Biometrika 36, 353-369.

Hall, P., 1987. Edgeworth expansion for Student's $t$ statistic under minimal moment conditions. Ann. Prob. 15, 920-931.

Hall, P., 1992. The bootstrap and Edgeworth expansion. Springer Series in Statistics, New York.

Hsu, P. L., 1945. The approximate distributions of the mean and variance of a sample of independent variables. Ann. Math. Stat. 16, 1-29.

Lehmann, E. L., Romano, J. P., 2005. Testing statistical hypotheses. 3rd ed. Springer Texts in Statistics, Springer, New York.

Wallace, D. L., 1958. Asymptotic approximations to distributions. Ann. Math. Stat. 29, 635-654. 
A. Corrected derivatives $g^{(i)}(0), i=1, \ldots, 4$

$$
\begin{aligned}
g^{(1)}(0) & =\frac{1}{2} z\left(\alpha_{4}-1\right)^{1 / 2} x \\
g^{(2)}(0) & =-z^{3}-\frac{1}{4} z\left(\alpha_{4}-1\right) x^{2} \\
g^{(3)}(0) & =-\frac{3}{2} z^{3}\left(\alpha_{4}-1\right)^{1 / 2} x+\frac{3}{8} z\left(\alpha_{4}-1\right)^{3 / 2} x^{3} \\
g^{(4)}(0) & =9 z^{5}+\frac{3}{2} z^{3}\left(\alpha_{4}-1\right) x^{2}-\frac{15}{16} z\left(\alpha_{4}-1\right)^{2} x^{4} .
\end{aligned}
$$

\section{B. Polynomials $q_{3}$ and $q_{4}$}

Theorem B.1. The polynomials $q_{3}$ and $q_{4}$ appearing in the Edgeworth expansion (1) are given by

$$
\begin{aligned}
q_{3}(y)= & \left(-\frac{1}{36} y^{6}+\frac{5}{24} y^{4}+\frac{5}{8} y^{2}+\frac{5}{48}\right) \alpha_{3} \alpha_{4}-\left(\frac{1}{20} y^{4}+\frac{1}{5} y^{2}+\frac{1}{40}\right) \alpha_{5} \\
& +\left(\frac{1}{162} y^{8}+\frac{7}{324} y^{6}-\frac{35}{216} y^{4}-\frac{175}{432} y^{2}-\frac{35}{432}\right) \alpha_{3}{ }^{3} \\
& +\left(\frac{1}{6} y^{6}-\frac{1}{4} y^{4}-\frac{1}{8} y^{2}-\frac{1}{16}\right) \alpha_{3},
\end{aligned}
$$




$$
\begin{aligned}
q_{4}(y)= & \left(-\frac{1}{1944} y^{11}-\frac{5}{1944} y^{9}+\frac{5}{108} y^{7}+\frac{25}{108} y^{5}-\frac{5}{216} y^{3}-\frac{35}{72} y\right) \alpha_{3}{ }^{4} \\
& +\left(\frac{1}{216} y^{9}-\frac{1}{18} y^{7}-\frac{5}{12} y^{5}+\frac{1}{6} y^{3}+\frac{29}{24} y\right) \alpha_{3}^{2} \alpha_{4} \\
& -\left(\frac{1}{36} y^{9}-\frac{1}{12} y^{7}-\frac{11}{36} y^{5}-\frac{1}{36} y^{3}+\frac{1}{6} y\right) \alpha_{3}{ }^{2} \\
& +\left(\frac{1}{60} y^{7}+\frac{2}{15} y^{5}-\frac{1}{12} y^{3}-\frac{1}{2} y\right) \alpha_{3} \alpha_{5} \\
& -\left(\frac{1}{288} y^{7}-\frac{7}{96} y^{5}+\frac{11}{96} y^{3}+\frac{37}{96} y\right) \alpha_{4}{ }^{2} \\
& +\left(\frac{1}{24} y^{7}-\frac{1}{4} y^{5}+\frac{1}{24} y^{3}+\frac{1}{4} y\right) \alpha_{4} \\
& -\left(\frac{1}{45} y^{5}-\frac{1}{18} y^{3}-\frac{1}{6} y\right) \alpha_{6}-\frac{1}{8} y^{7}+\frac{3}{8} y^{5} .
\end{aligned}
$$

C. Asymptotic expansions of $b_{n}, h_{n}(t)$ and $g_{n}(t)$

Lemma C.1. Setting $\lambda=n^{-1 / 2}$, asymptotic expansions of $b_{n}, h_{n}(t)$ and $g_{n}(t)$ are given by

$$
\begin{aligned}
b_{n}=1+ & \left(C_{1} / 2\right) \lambda+\left(C_{2} / 2+3 C_{1}^{2} / 8\right) \lambda^{2} \\
+ & {\left[C_{3} / 2+C_{1} C_{2}+9 C_{1}^{3} / 16-C_{1}\left(C_{2}+C_{1}{ }^{2}\right) / 4\right] \lambda^{3} } \\
+ & {\left[C_{4} / 2+C_{1} C_{3}+C_{2}{ }^{2} / 2+3 C_{2} C_{1}{ }^{2} / 2-C_{1}\left(C_{3}+2 C_{1} C_{2}+C_{1}^{3}\right) / 8\right.} \\
& -\left(C_{2}+C_{1}{ }^{2}\right)^{2} / 8-C_{1}\left(C_{3} / 2+C_{1} C_{2}+C_{1}^{3} / 2\right) / 4+59 C_{1}{ }^{4} / 128 \\
& \left.+3 C_{1}{ }^{2}\left(C_{2}+C_{1}{ }^{2}\right) / 16\right] \lambda^{4}+O\left(\lambda^{5}\right),
\end{aligned}
$$




$$
\begin{aligned}
h_{n}(t)= & \left(C_{1} t / 2\right) \lambda+(t / 8)\left(4 C_{2}+3 C_{1}{ }^{2}-C_{1}{ }^{2} t^{2}\right) \lambda^{2} \\
+ & (t / 48)\left(24 C_{3}+36 C_{1} C_{2}+15 C_{1}^{3}-12 t^{2} C_{1} C_{2}-10 t^{2} C_{1}{ }^{3}+t^{4} C_{1}{ }^{3}\right) \lambda^{3} \\
- & (t / 384)\left(-192 C_{4}-288 C_{1} C_{3}-144 C_{2}{ }^{2}-360 C_{1}{ }^{2} C_{2}-105 C_{1}{ }^{4}\right. \\
& +96 t^{2} C_{1} C_{3}+240 t^{2} C_{1}{ }^{2} C_{2}+105 t^{2} C_{1}{ }^{4}+48 t^{2} C_{2}{ }^{2} \\
& \left.-24 t^{4} C_{1}{ }^{2} C_{2}-21 t^{4} C_{1}{ }^{4}+t^{6} C_{1}{ }^{4}\right) \lambda^{4}+O\left(\lambda^{5}\right), \\
g_{n}(t)= & 1-\left(C_{1} t^{2} / 2\right) \lambda-\left(C_{1}{ }^{2} t^{2} / 2+t^{2} C_{2} / 2-t^{4} C_{1}{ }^{2} / 8\right) \lambda^{2} \\
- & \left(t^{2} C_{1} C_{2}+t^{2} C_{1}^{3} / 2+t^{2} C_{3} / 2-t^{4} C_{1}^{3} / 4-t^{4} C_{1} C_{2} / 4+t^{6} C_{1}^{3} / 48\right) \lambda^{3} \\
+ & \left(t^{4} C_{2}{ }^{2} / 8+t^{8} C_{1}{ }^{4} / 384-t^{2} C_{2}{ }^{2} / 2-t^{2} C_{1}^{4} / 2-t^{6} C_{1}{ }^{4} / 16+3 t^{4} C_{1}{ }^{4} / 8\right. \\
& \left.-t^{6} C_{1}{ }^{2} C_{2} / 16+t^{4} C_{1} C_{3} / 4-t^{2} C_{1} C_{3}-3 t^{2} C_{1}{ }^{2} C_{2} / 2+3 t^{4} C_{1}{ }^{2} C_{2} / 4-t^{2} C_{4} / 2\right) \lambda^{4} \\
+ & O\left(\lambda^{5}\right) .
\end{aligned}
$$

